Participant Flow:

9 participants were recruited
9 participants were enrolled into the study
9 participants were interviewd 


\section{Baseline Characteristics:}

As this was a qualitative study, specific demographics were not required, as it was more important to continue to recruit and interview until thematic saturation was reached. Each of the participants had presented to another Health Care Professional with late GI symptoms following pelvic radiotherapy.

The following table shows participants characteristics:

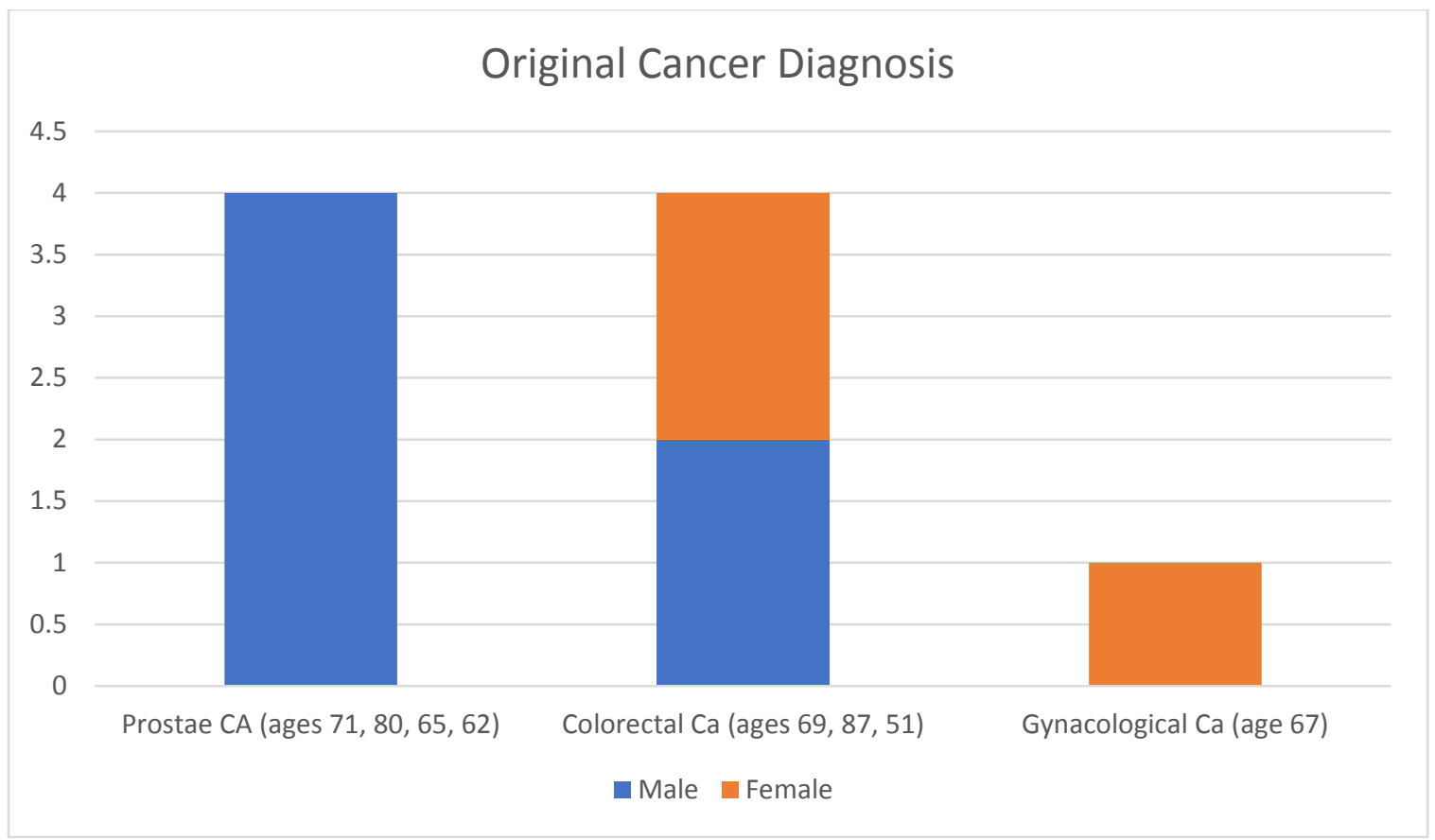




\section{Outcome Measures:}

As a qualitative study, my findings were grouped into three main areas of experience. There was found to be a significant element of stigma experienced by all the participants. Each of them also had negative experiences with healthcare providers over accessing help for their late effect symptoms, and they all commented how important support was to them after their cancer treatment had finished, and that this support often stopped when the initial cancer treatment stopped. The full study presents narrative extracts of the participants experiences and these stories have been presented at meetings and conferences as posters. 
Adverse Events:

There were no adverse events associated with this trial. 